\title{
Correction to: Contextualizing Math-Related Strengths and Math Achievement: Positive Math Orientations, Social Supports and the Moderating Effects of Prior Math Knowledge
}

\section{Krystal L. Williams ${ }^{1}$}

Published online: 26 April 2021

(C) Springer Nature Switzerland AG 2021

\section{Correction to: Journal for STEM Educ Res https://doi.org/10.1007/s41979-020-00033-z}

In this paper, 2 equations displayed incorrectly. The correct equations are presented below:

(1) $\quad 1^{\text {th }}$ Grade Math Knowledge $=\beta_{0}+\beta_{1}$ Prior Math Achievement $+\beta_{2}$ Math ID $+\beta_{3}$ Math SE $+\beta_{4}$ Math Int $+\beta_{5}$ Math Util $+\beta_{6}$ Gen Math Support

$+\beta_{7}$ Math Related Social Groups $+\beta_{8}$ Female $+\beta_{9}$ Underrep SOC $+\beta_{10}$ Multi Racial $+\beta_{11} \mathrm{SES}+\varepsilon$

(2) $\quad \mathbf{1 1}^{\text {th }}$ Grade Math Knowledge $=\beta_{0}+\beta_{1}$ Prior Math Achievement $+\beta_{2}$ Math ID $+\beta_{3}$ Math SE $+\beta_{4}$ Math Int $+\beta_{5}$ Math Util $+\beta_{6}$ Gen Math Support

$+\beta_{7}$ Math Related Social Groups $+\beta_{8}$ Female $+\beta_{9}$ Underrep SOC $+\beta_{10}$ Multi Racial $+\beta_{11}$ SES $+\beta_{12}$ MLS Measure $\times$ Prior Math Achievement $+\varepsilon$

where MLS Measure=Math ID, Math SE, Math Int, Math Util, Gen Math Support, or Math-Related Social Groups

The online version of the original article can be found at https://doi.org/10.1007/s41979-020-00033-z

Krystal L. Williams

Krystal.L.Williams@ua.edu

1 University of Alabama, Tuscaloosa, AL, USA 